\title{
Diabetic Cardiac Autonomic Dysfunction and Its Relationship with Duration of Diabetes
}

\author{
Phurpa $^{1}$, Sultana Ferdousi ${ }^{2}$
}

\begin{abstract}
Background: Duration of diabetes may have an important influence on cardiac autonomic dysfunction in type 2 diabetes mellitus. Objective: To assess the relationship of cardiac autonomic dysfunction in type 2 diabetes mellitus(T2DM) with the duration of diabetes. Methods: This crosssectional study was carried out from January to December 2015 on 54 long-term T2DM male patients aged 45 to 55 years, selected from the Endocrinology OPD of BSMMU, Dhaka. Thirty age matched recently diagnosed male T2DM patients(RT2DM) were control. Cardiac autonomic function was assessed by Heart Rate Variability(HRV). HRV measures were obtained by RMS multi channel polyrite-D. For statistical analysis, unpaired t-test and Pearson's correlation coefficient test were used. Results: Mean RR, SDNN, and HF nu were significantly lower $(p<0.001)$ whereas, mean HR, LF nu and LF/HF were significantly $(\mathrm{p}<0.001)$ higher in LT2DM than those of RT2DM. SDNN, RMSSD and variance showed significant $(\mathrm{p}<0.001)$ negative correlation with the duration of diabetes. Conclusions: Duration of diabetes had strong negative relationship with the diabetic cardiac autonomic dysfunction.
\end{abstract}

Key words: HRV, SDNN, T2DM

\author{
J Bangladesh Soc Physiol. 2016, June; 11(1): 1-5 \\ For Authors Affiliation, see end of text. \\ http://www.banglajol.info/index.php/JBSP
}

\section{Introduction}

he natural course of cardiac autonomic
neuropathy (CAN), a dreadful
complication of T2DM, is still

unclear $^{1}$. However, initially it presents as subclinical form and turns into clinical form as the disease progresses ${ }^{1}$. The subclinical CAN does not manifest any symptoms of CAN and appear normal in Ewing battery test ${ }^{1-3}$; but, it can be detected through abnormalities in HRV 4 . The cardiac autonomic dysfunction in subclinical CAN is essentially characterized by greater parasympathetic nerve function impairment with the consequent sympathetic predominance, leading to the development of autonomic imbalance ${ }^{1}$.

Received 20 Nov. 2015; Accepted 4th March 2016
HRV provides a quantitative measurement of autonomic nerve function of individual branches of autonomic nervous system (ANS) and its balance $^{5}$. Reduced HRV is the earliest sign of $\mathrm{CAN}^{2,5-8}$. Decline in HRV in T2DM was seen in several studies ${ }^{3,9-12}$. Despite the unclear nature of association between the duration of diabetes and autonomic dysfunction ${ }^{13}$, many studies observed the existence of relationship between these two ${ }^{3,7,9,14-15}$. The aim of this study was to assess the impact of the duration of diabetes on the cardiac autonomic dysfunction in type 2 diabetic patients without evidence of CAN based on Ewing battery test.

\section{Methods:}

This cross-sectional study was conducted from January, 2015 to December, 2015 at the 
Department of Physiology, Bangabandhu Sheikh Mujib Medical University (BSMMU), Dhaka. The protocol of this study was approved by the Institutional Review Board of BSMMU. For this study, total 84 CAN asymptomatic and negative by Ewing battery test diagnosed male patients of T2DM following criteria of $\mathrm{WHO}^{16}$ (age: 45 - 55 years; HbA1c: 5 - $10 \%$ ) were recruited from the Endocrinology Out Patient Department,BSMMU, after taking written informed consent. All these patients were only under oral hypoglycemic agents in addition to lifestyle modifications. Thirty recently diagnosed $(<6$ months duration) T2DM (RT2DM) constituted controls and 54 ( 5 to 10 years of duration of DM) long term T2DM (LT2DM) patients formed study group. The patients who had history or were then suffering from diabetic retinopathy, nephropathy (creatinine $>2 \mathrm{mg} / \mathrm{dl}$ ), hypertension (>140/90 $\mathrm{mm} \mathrm{Hg}$ ), coronary artery diseases, epilepsy, migraine, psychiatric disorders, respiratory disorders, hypo- and hyperthyroidism, consuming drugs with effect on autonomic nervous system function or who were current smokers, yoga practitioners and athletes were excluded.

A thorough physical examination and Ewing battery test were done to the selected patients in the Autonomic Nerve Function Lab, Department of Physiology, BSMMU. The patients who had normal findings in Ewing battery test subsequently underwent tests for fasting plasma glucose (FPG), 2-h plasma glucose (2-h PG), hemoglobin A1c (HbA1c) and plasma creatinine to see the glycemic status and to rule out nephropathy.

The finally selected patients were instructed to finish their meals by 09:00 PM, have sound sleep, avoid physical and mental stress, and refrain from drugs having effect on central nervous system, in the night prior to the HRV test. The following morning they took light breakfast without coffee or tea. For HRV measurement, the room temperature of the Autonomic Lab was set around $25-28 \mathrm{C}$ and the patient was seated in the lab for 15 minutes before HRV recording allowing him to adjust with the lab environment. After that, the patient lay on the examination bed in supine position and took rest for 5 minutes. Then, ECG was recorded on lead II for 5 minutes, by RMS multichannel polyrite-D (India), from which measures of HRV was analyzed by RMS polyrite software.

Data were expressed as mean and SE. SPSS for Windows, Version 16.0 was used for statistical analysis. Independent sample t test was used to compare the mean values of RT2DM and LT2DM group. Pearson's Correlation Coefficient test was done to analyze the linear correlation between the duration of diabetes and HRV parameters. p value $<0.05$ was taken as statistically significant level.

\section{Results}

General characteristics of all subjects are presented in Table I. long term diabetic group had significantly higher mean pulse rate, two hour post pandial plasma glucose and glycosylated hemoglobin level than that of recently diagnosed diabetics. Mean RR, SDNN, and HF nu were significantly $(\mathrm{p}<0.001)$ lower, whereas, mean $\mathrm{HR}, \mathrm{LF}$ nu and LF/HF were significantly higher $(\mathrm{p}<0.001)$ in T2DM group compared to diabetics of short duration(Table II). On correlation analysis, SDNN, variance and RMSSD showed significant $(p<0.001)$ negative correlation with the duration of diabetes in long term diabetic patients (Figure 1,2,3) whereas, mean RR, mean HR and frequency domain parameters showed no significant association with duration and these data were not shown. 
Table I : General Characteristics of All Subjects $(\mathrm{n}=84)$

\begin{tabular}{lcc}
\hline Variables & RT2DM $(\mathrm{n}=30)$ & LT2DM $(\mathrm{n}=54)$ \\
\hline Age $($ years $)$ & $48.54 \pm 0.60$ & $49.78 \pm 0.38$ \\
BMI $\left(\mathrm{Kg} / \mathrm{m}^{2}\right)$ & $25.03 \pm 0.74$ & $23.17 \pm 0.19$ \\
Duration of diabetes $(\mathrm{yrs})$ & $0.31 \pm 0.42$ & $6.5 \pm 0.18^{* * *}$ \\
Pulse rate (beats/min) & $77.69 \pm 0.73$ & $80.48 \pm 0.58^{*}$ \\
SBP $(\mathrm{mm} \mathrm{Hg})$ & $125.82 \pm 1.54$ & $126.02 \pm 1.12$ \\
DBP $(\mathrm{mm} \mathrm{Hg})$ & $78.65 \pm 0.81$ & $78.61 \pm 0.69$ \\
FPG $(\mathrm{mmol} / \mathrm{L})$ & $8.16 \pm 0.26$ & $7.67 \pm 0.14$ \\
2-h PG $(\mathrm{mmol} / \mathrm{L})$ & $11.30 \pm 0.31$ & $12.30 \pm 0.23^{*}$ \\
HbA1c $(\%)$ & $8.11 \pm 0.21^{ \pm}$ & $7.18 \pm 0.07^{* * *}$ \\
\hline
\end{tabular}

Data were expressed as mean \pm SE. Statistical analysis was done by unpaired t test. RT2DM: Recently diagnosed type 2 diabetic patients ( $<6$ months duration); LT2DM: Long term type 2 diabetic patients ( 5 to 10 years duration) ;BMI: body mass index; SBP: systolic blood pressure; DBP: diastolic blood pressure; FPG:Fasting plasma glucose;2-hPG:2 hour post pandial glucose; HbA1c: Glycosylated hemoglobin ***p $<0.001{ }^{*}$ p value $<0.05$

Table II : HRV measures of recently diagnosed and long term type 2 diabetic patients.(n=84)

\begin{tabular}{lcc}
\hline Parameters & RT2DM $(\mathrm{n}=30)$ & LT2DM $(\mathrm{n}=54)$ \\
\hline HR (beat/min) & $67.23 \pm 1.77$ & $79.65 \pm 1.13^{* * *}$ \\
R-R(sec) & $0.90 \pm 0.11$ & $0.76 \pm 0.08^{* * *}$ \\
SDNN(ms) & $45.01 \pm 2.53$ & $28.75 \pm 1.09^{*}$ \\
LF (n.u.) & $52.99 \pm 2.79$ & $66.03 \pm 1.48^{* * *}$ \\
HF (n.u.) & $47.01 \pm 2.79$ & $33.97 \pm 1.48^{* * *}$ \\
LF/HF & $1.38 \pm 0.17$ & $2.19 \pm 0.84^{* * *}$ \\
\hline
\end{tabular}

Data were expressed as Mean \pm SE. Statistical analysis were done by Independent sample t-test. HR: Heart rate, R-R: Interval between two successive QRS complex; SDNN: Standard deviations of all normal RR intervals; RT2DM: Recently diagnosed type 2 diabetic patients ( $<6$ months duration); LT2DM: Long term type 2 diabetic patients (5 to 10 years duration). ${ }^{* * *} \mathrm{p}<0.001,{ }^{*} \mathrm{p}<0.05$

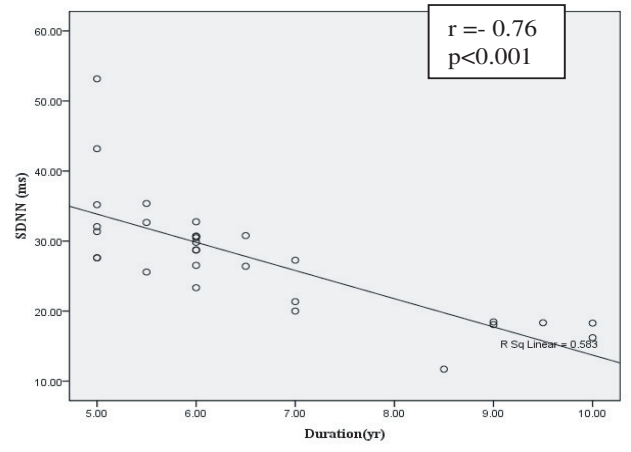

Figure 1: Correlation of SDNN showed significant negative correlation with duration of diabetes in LT2DM.

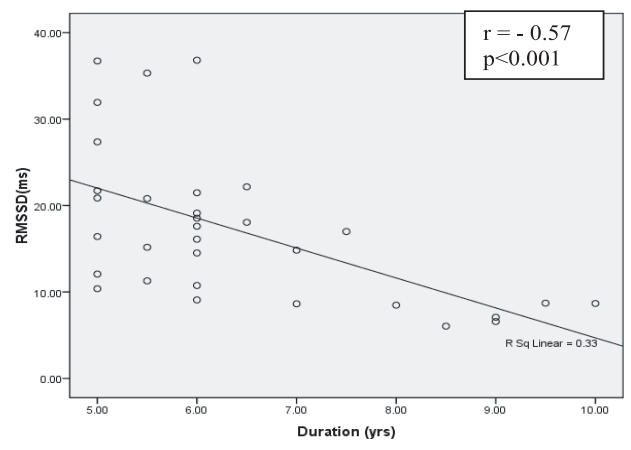

Figure 2: Correlation of RMSSD showed significant negative correlation with duration of diabetes in LT2DM. 


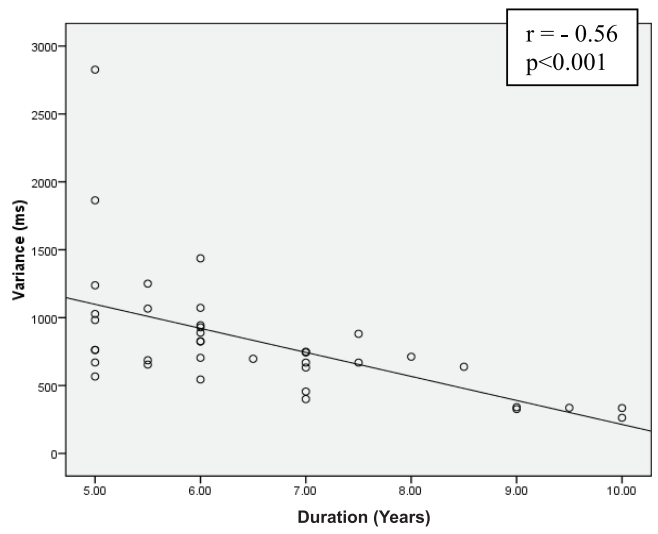

Figure 3: Correlation of variance showed significant negative correlation with duration of diabetes in LT2DM.

\section{Discussion}

In this study relationship between the autonomic dysfunction assessed by short-term HRV, giving reliable quantitative measurement of resting cardiac autonomic modulation ${ }^{5}$, and the disease duration was explored in long term type 2 diabetic patients . This study found significant decline in mean RR, SDNN, HF nu and significant increase in mean HR, LF nu and LF/ HF in diabetic patients with longer duration of diabetes compared to diabetics with short duration and also without evidence of CAN. These results suggest deterioration of parasympathetic function and shifting of autonomic balance towards higher sympathetic activity as the duration of diabetes advances. Similar findings were reported by other studies $^{1,3,12}$. This finding corroborates the observation of significant decrease in HRV, 5 10 years after diagnosis of T2DM made by Tarveinenet al. ${ }^{3}$

On further analysis, value of SDNN, a reflection of overall variability of heart rate, variance and RMSSD, marker of parasympathetic nerve activity exhibited strong negative correlation with the duration of DM. Similar associations were observed in previous studies ${ }^{3,7}$. This might be due to parasympathetic nerve dysfunction, which occurs within $5-10$ years after diagnosis of $\mathrm{T}_{2} \mathrm{DM}^{3}$. However, similar to previous study ${ }^{3}$, no significant linear association was found with mean RR, mean HR, LF nu, HF nu and LF/HF. This might be because our patients were of 5 10 years of duration, and during this period the sympathetic modulation is not compromised significantly, it rather happens after $10-15$ years after diagnosis of $\mathrm{T} 2 \mathrm{DM}^{3}$. This finding agrees the statement that 'vagus is the first to be affected during the initial stage of CAN and later sympathetic denervation ensues' made by other studies ${ }^{1,9,17}$. But on the contrary, Hsiao et al. ${ }^{9}$ stated that sympathetic nerve damage might occur earlier than parasympathetic nerve damage.

Duration of diabetes is an important factor which influences on the development of diabetic cardiac autonomic neuropathy ${ }^{6}$. Cardiac autonomic dysfunction or subclinical CAN could be even present at the time or within a year of diagnosis of T2 $\mathrm{DM}^{16,17}$. Many studies observed association between cardiac autonomic dysfunction with disease duration $3,7,9,14-15$, however, the nature of this relationship is still unclear ${ }^{13}$.Yet, the opposite finding was reported by Ko et al. ${ }^{18}$. The pathogenesis of CAN is still not well elucidated even though many researchers blame prolonged hyperglycemia induced oxidative and nitrosative stress, which in turn cause neuronal toxicity and death ${ }^{17,19-20}$. These above key observations of the present study, clearly delineated the negative impact of duration of disease on cardiac autonomic function leading towards its dysfunction with the inevitable consequence of diabetic autonomic damage if left untreated.

\section{Conclusions}

Cardiac autonomic dysfunction, especially impairment of parasympathetic modulation, in T2DM patients without apparent CAN showed strong relationship with the duration of diabetes. 
This suggests that the parasympathetic nerve dysfunction occurs earlier than sympathetic nerve denervation.

\section{Conflict of interest: None}

\section{Acknowledgement}

The authors of this work acknowledge the partial financial support from the research grant by University grant commission of Bangladesh.

\section{Author affiliations}

1. Phurpa, MD resident, Dept of Physiology,Bangabandhu Sheikh Mujib, Medical University,Shahbag,Dhaka email:pgyd@gmail.com

2. Sultana Ferdousi, Associate Professor, Dept of Physiology, Bangabandhu Sheikh Mujib, Medical University,Shahbag, Dhaka email:sferdousiratna @gmail.com; sferdousiratna@yahoo.com

\section{References}

1. Dimitropoulos G, Tahrani AA, Stevens MJ. Cardiac Autonomic Neuropathy in Patients with Diabetes Mellitus. World J Diabetes. 2014; 5(1): 17-39.

2. Khandoker AH, Jelinek HF, Moritanic T, Palaniswamia M. Association of cardiac autonomic neuropathy with alteration of sympatho-vagal balance through heart rate variability analysis. Med EngPhy. 2010; 32: 161-167.

3. Tarvainen MP, Laitinen TP, Lipponen JA, Cornforth DJ, Jelinek HF. Cardiac autonomic dysfunction in type 2 diabetes - effect of hyperglycemia and disease duration. FrotierEndocrinol. 2014; 5: 1-9.

4. Consensus Statement. Standardized Measures in Diabetic Neuropathy. Diabetes Care. 1995; 18: 59-82.

5. Task Force of the European Society of Cardiology and the North American Society of pacing and electrophysiology. Heart Rate Variability: Standards of Measurement, Physiological Interpretation and Clinical Use. 1996; Circulation. 93: 1043- 65.

6. Ziegler D. Diabetic Cardiovascular Autonomic Neuropathy: Prognosis, Diagnosis and Treatment. Diabetes Metab Rev. 1994; 10(4): 339-383.

7. Balcioglu S, Arslan U, Turkoglu S, Ozdemir M, Cengel A. Heart Rate Variability and Heart Rate Turbulence in Patients with Type 2 Diabetes Mellitus with Versus without Cardiac Autonomic Neuropathy. 2007; doi: 10.1016/j.amjcard.2007.03.106.

8. Pop-Bosui R. Cardiac Autonomic Neuropathy in Diabetes. Diabetes Care. 2010; 33(2): 434-441.

J Bangladesh Soc Physiol. 2016, June; 11(1): 1-5
9. Hsiao JY, Tien JY, Hsiao CT, Weng HH, Chung TC, Hsieh MC. The Relationship between Diabetic Autonomic Neuropathy and Diabetic Risk Factors in a Taiwanese Population. J Int Med Res. 2011; 39: 1155 -1162 .

10. Tale S, Sontakke TR. Time-Frequency Analysis of Heart Rate Variability Signal in prognosis of Type 2 Diabetic Autonomic Neuropathy. International Conference on Biomedical Engineering and Technology; Singapore, 2011.

11. Deepak A, Aithal K, Khode VH, Nallulwar SC. Short term heart rate variability for early assessment of autonomic neuropathy in patients with type 2 diabetes mellitus: A comparative cross-sectional study. Annals Nigerian Med. 2014; 8(1): 4-7.

12. Pramodh V, Kumar MP, Prasad BAK. Heart rate variability in type 2 diabetics. IJBR. 2014; 5(5): 364367.

13. Vinik AI, Ziegler D. Diabetic Cardiovascular Autonomic Neuropathy. Circulation. 2007; 115:387397.

14. Alam KM, Begum N, Molla HM, and Begum S. Sympathetic Nerve Function Status in Type 2 Diabetes Mellitus: Relationship with Glycaemic Status and Duration. Journal of Medical Sciences and Research. 2008; 11(1)23-30.

15. Spallone V, Ziegler D, Freeman R, Bernardi L, Frontoni S, Pop-Busui R, Stevens M, Kempler P, Hilsted J, Tesfaye S, Low P and Valensi P. Cardiovascular autonomic neuropathy in diabetes: clinical impact, assessment, diagnosis, and management. Diabetes Metab Res Rev. 2011; 27: 639-653.

16. World Health Organization. Definition and Diagnosis of Diabetes Mellitus and Intermediate Hyperglycemia: Report of a WHO/IDF Consultation. [Internet]. Geneva: World Health Organization; c2006. [Cited 2015 Mar 13]. Available from: https://www.idf.org.

17 Kuehl M, Stevens MJ. Cardiovascular autonomic neuropathies as complications of diabetes mellitus. Nat. Rev. Endocrinol. 2012; 8: 405-416.

18. Ko SH, Park SA, Cho JH, Song KH, Yoon KH, Cha BY, Son HY, Yoo KD, Moon KW, Park YM, Ahn YB, (2008). Progression of Cardiovascular Autonomic Dysfunction in Patients with Type 2 Diabetes. Diabetes Care. 31(9):1832-1836.

19. Giacco F, Brownlee M. Oxidative stress and diabetic complications. Circ Res. 2010; 107(9): 1058-1070.

20. Bandeira SM, da Fonseca LJS, da Silva Guedes G, Rabelo LA, Goulart MOF, Vasconcelos SML. Oxidative Stress as an Underlying Contributor in the Development of Chronic Complications in Diabetes Mellitus. Int. J. Mol. Sci. 2013; 14: 3265-3284. 